\title{
Finite Eulerian posets which are binomial or Sheffer
}

\author{
Hoda Bidkhori
}

North Carolina State University, NC, USA

\begin{abstract}
In this paper we study finite Eulerian posets which are binomial or Sheffer. These important classes of posets are related to the theory of generating functions and to geometry. The results of this paper are organized as follows: (1) We completely determine the structure of Eulerian binomial posets and, as a conclusion, we are able to classify factorial functions of Eulerian binomial posets; (2) We give an almost complete classification of factorial functions of Eulerian Sheffer posets by dividing the original question into several cases; (3) In most cases above, we completely determine the structure of Eulerian Sheffer posets, a result stronger than just classifying factorial functions of these Eulerian Sheffer posets. We also study Eulerian triangular posets. This paper answers questions posed by R. Ehrenborg and M. Readdy. This research is also motivated by the work of R. Stanley about recognizing the boolean lattice by looking at smaller intervals.

Résumé. Nous étudions les ensembles partiellement ordonnés finis (EPO) qui sont soit binomiaux soit de type Sheffer (deux notions reliées aux séries génératrices et à la géométrie). Nos résultats sont les suivants: (1) nous déterminons la structure des EPO Euleriens et binomiaux; nous classifions ainsi les fonctions factorielles de tous ces EPO; (2) nous donnons une classification presque complète des fonctions factorielles des EPO Euleriens de type Sheffer; (3) dans la plupart de ces cas, nous déterminons complètement la structure des EPO Euleriens et Sheffer, ce qui est plus fort que classifier leurs fonctions factorielles. Nous étudions aussi les EPO Euleriens triangulaires. Cet article répond à des questions de R. Ehrenborg and M. Readdy. Il est aussi motivé par le travail de R. Stanley sur la reconnaissance du treillis booléen via l'étude des petits intervalles.
\end{abstract}

Keywords: Eulerian poset, binomial poset, Sheffer poset

\section{Introduction}

There are many theories which unify various aspects of enumerative combinatorics and generating functions. One such successful theory introduced by Doubilet, Rota and Stanley [3] is that of binomial posets. Classically, binomial posets are infinite posets with the property that every two intervals of the same length have the same number of maximal chains. Doubilet, Rota and Stanley show this chain regularity condition gives rise to universal families of generating functions. Ehrenborg and Readdy [5] and Reiner [9] independently generalized the notion of binomial posets to a larger class of posets called Sheffer posets or upper binomial posets.

Ehrenborg and Readdy [4] gave a complete classification of the factorial functions of infinite Eulerian binomial posets and infinite Eulerian Sheffer posets. Recall that infinite posets are those posets which 
contain an infinite chain. They posed the open question of characterizing the finite case. This paper deals with these questions.

A Sheffer poset is a graded poset such that the number of maximal chains $D(n)$ in an $n$-interval $[\hat{0}, y]$ depends only on $\rho(y)=n$, the rank of the element $y$, and the number $B(n)$ of maximal chains in an $n$ interval $[x, y]$, where $x \neq \hat{0}$, depends only on $\rho(x, y)=\rho(y)-\rho(x)$. The two functions $B(n)$ and $D(n)$ are called the binomial factorial function and Sheffer factorial function, respectively. Binomial posets are a special class of Sheffer posets. A binomial poset is a graded poset such that the number of maximal chains $B(n)$ in an $n$-interval $[x, y]$ depends only on $\rho(x, y)=\rho(y)-\rho(x)$.

Binomial posets were previously considered in [1], [3], [8], [11] and [13]. Ehrenborg and Readdy [5] used Sheffer posets and a generalization of $R$-labeling to study augmented $r$-signed permutations. Reiner [9] used them to derive generating functions counting signed permutations by two statistics.

A graded poset $P$ is Eulerian if every non-singleton interval of $P$ satisfies the Euler-Poincaré relation. (See Definition 2.1) Eulerian posets form an important class of posets as there are many geometric examples such as the face lattices of convex polytopes, and more generally, the face posets of regular CW-spheres.

As we mentioned above, Ehrenborg and Readdy in [4] classify the factorial functions of infinite Eulerian binomial posets and infinite Eulerian Sheffer posets. Since we are concerned here with finite posets, we drop the requirement that binomial, Sheffer and triangular posets have an infinite chain. This paper studies the following natural questions, as suggested by Ehrenborg and Readdy in [4].

1. Which Eulerian posets are binomial?

2. Which Eulerian posets are Sheffer?

Stanley has proved that one can recognize boolean lattices by looking at smaller intervals (see [7], Lemma 8). Farley and Schmidt answer a similar question for distributive lattices in [6]. The project of studying Eulerian binomial posets and Eulerian Sheffer posets is also motivated by their work. In many cases we use the factorial function of smaller intervals to characterize the whole poset.

\subsection{Acknowledgments}

The author would like to thank Richard Stanley for suggesting this research problem. I also would like to thank Richard Ehrenborg, Margaret A. Readdy and Richard Stanley for helpful discussions and comments and thank Craig Desjardins for reading a draft of this paper. I am grateful to two anonymous referees for many helpful suggestions..

\section{Background and Definition}

We encourage readers to consult Chapter 3 of [12] for basic poset terminology. All the posets which are considered in this paper are finite.

We begin by recalling that a graded interval satisfies the Euler-Poincaré relation if it has the same number of elements of even rank as of odd rank.

Definition 2.1. 1. A graded poset is Eulerian if every non-singleton interval satisfies the Euler-Poincaré relation. Equivalently, a poset $P$ is Eulerian if its Möbius function satisfies $\mu(x, y)=(-1)^{\rho(x, y)}$ for all $x \leq y$ in $P$, where $\rho$ denotes the rank function of $P$. 
2. Consider a graded poset $P$ with rank function $\rho$. If $\rho(x, y)=n$, then we call $[x, y]$ an $n$-interval.

Definition 2.2. A finite graded poset $P$ with unique minimal element $\hat{0}$ and unique maximal element $\hat{1}$ is called $a$ (finite) binomial poset if it satisfies the following condition:

1. For all $n \in \mathbb{N}, n \leq \operatorname{rank}(P)$, any two $n$-intervals have the same number $B(n)$ of maximal chains. We call $B(n)$ the factorial function or binomial factorial function of the poset $P$.

Definition 2.3. A finite graded poset $P$ with a unique minimal element $\hat{0}$ and a unique maximal element $\hat{1}$ is called a (finite) Sheffer poset if it satisfies the following two conditions:

1. Any pair of $n$-intervals $[\hat{0}, y]$ and $[\hat{0}, v]$ have the same number $D(n)$ of maximal chains.

2. Any pair of $n$-intervals $[x, y]$ and $[u, v]$ such that $x \neq \hat{0}$ and $u \neq \hat{0}$ have the same number $B(n)$ of maximal chains.

Let us consider a Sheffer poset $P$. An interval $[\hat{0}, y]$, where $y \neq \hat{0}$, is called a Sheffer interval whereas an interval $[x, y]$ with $x \neq \hat{0}$ is called a binomial interval. The functions $B(n)$ and $D(n)$ are called the binomial factorial function and Sheffer factorial function of $P$, respectively. Next we define $A(n)$ and $C(n)$ to be the number of coatoms in a binomial interval of length $n$, respectively, a Sheffer interval of length $n$. The functions $A(n)$ and $C(n)$ are called the atom function and coatom function of $P$, respectively. The number of elements of rank $k$ in a Sheffer interval of rank $n$ is

$$
\frac{D(n)}{D(k) B(n-k)} \text {. }
$$

Moreover, for a binomial interval $[x, y]$ of rank $n$ in a Sheffer poset, the number of elements of rank $k$ is equal to

$$
\frac{B(n)}{B(k) B(n-k)} .
$$

The dual suspension of a poset $P$ is defined in [4] as follows.

Definition 2.4. Let $P$ be a poset with $\hat{0}$. We define the dual suspension of $P$, denoted $\Sigma^{*}(P)$, to be the poset $P$ with two new elements $a_{1}$ and $a_{2}$. $\Sigma^{*}(P)$ has the following order relation: $\hat{0}<_{\Sigma^{*}(P)} a_{i}<_{\Sigma^{*}(P)}$ $y$, for all $y>\hat{0}$ in $P$ and $i=1,2$. That is, the elements $a_{1}$ and $a_{2}$ are inserted between $\hat{0}$ and atoms of $P$. Clearly if $P$ is Eulerian then so is $\Sigma^{*}(P)$. Moreover, if $P$ is a binomial poset then $\Sigma^{*}(P)$ is a Sheffer poset with the factorial function $D_{\Sigma^{*}(P)}(n)=2 B(n-1)$, for $n \geq 2$.

Definition 2.5. Let $P$ be a poset with $\hat{1}$. We define the suspension of $P$, denoted by $\Sigma(P)$, to be the poset $P$ with two new elements $a_{1}$ and $a_{2}$ adjoined with the additional order relations that $y<_{\Sigma(P)} a_{i}<_{\Sigma(P)} \hat{1}$, for all $y<\hat{1}$ in $P$ and $i=1,2$.

The dual of the poset $P$, denoted $P^{*}$, is defined as follows: $P^{*}$ has the same set of elements as $P$ and the following order relation: $x<_{P^{*}} y$ if and only if $y<_{P} x$.

Definition 2.6. The boolean lattice $B_{n}$ of rank $n$ is the poset of subsets of $[n]=\{1, \ldots, n\}$ ordered by inclusion.

Definition 2.7. The butterfly poset $T_{n}$ of rank $n$ consists of the elements of $\{\hat{0}\} \cup\left(D_{n-1} \times\{1,2\}\right) \cup\{\hat{1}\}$, where $D_{n-1} \times\{1,2\}$ is the direct product of the chain of length $n-1$, denoted by $D_{n-1}$, and the antichain of rank 2 , with the order relation $(k, i) \prec(k+1, j)$ for all $i, j \in\{1,2\}$. Also $\hat{0}$ and $\hat{1}$ are the unique minimal and maximal elements of this poset, respectively. Clearly, $T_{n} \cong \Sigma^{*}\left(T_{n-1}\right)$. 


\section{Finite Eulerian binomial posets}

In this section, we classify the structure of finite Eulerian binomial posets.

First we provide some examples of finite binomial posets. See [4] for infinite versions of Examples 3.1 and 3.2

Example 3.1. The boolean lattice $B_{n}$ of rank $n$ is an Eulerian binomial poset with factorial function $B(k)=k !$ and atom function $A(k)=k, k \leq n$. Every interval of length $k$ of this poset is isomorphic to $B_{k}$.

Example 3.2. The butterfly poset $T_{n}$ of rank $n$ is an Eulerian binomial poset with factorial function $B(k)=2^{k-1}$ for $1 \leq k \leq n$ and atom function $A(k)=2$, for $2 \leq k \leq n$, and $A(1)=1$.

It is not hard to see that in any $n$-interval of an Eulerian binomial poset $P$ with factorial function $B(k)$ for $1 \leq k \leq n$, the Euler-Poincaré relation is stated as follows:

$$
\sum_{k=0}^{n}(-1)^{k} \cdot \frac{B(n)}{B(k) B(n-k)}=0 .
$$

The following is [4, Lemma 2.6].

Lemma 3.3. Let $P$ be a graded poset of odd rank such that every proper interval of $P$ is Eulerian. Then $P$ is an Eulerian poset.

Lemma 3.4. Let $P$ be an Eulerian binomial poset of rank 3. Then the poset $P$ and its factorial function $B(n)$ satisfy the following conditions:

(i) $B(2)=2$ and $B(3)=2 q$, where $q$ is a positive integer such that $q \geq 2$.

(ii) There is a list of integers $q_{1}, \ldots, q_{r}, q_{i} \geq 2$, such that $P \cong \boxplus_{i=1, \ldots, r} P_{q_{i}}$, where $P_{q_{i}}$ is the face lattice of the $q_{i}$-gon.

This result is [4, Example 2.5].

R. Ehrenborg and M. Readdy proved the following two propositions. See [4, Lemma 2.17 and Prop. 2.15].

Proposition 3.5. Let $P$ be a binomial poset of rank $n$ with factorial function $B(k)=2^{k-1}$ for $1 \leq k \leq n$. Then the poset $P$ is isomorphic to the butterfly poset $T_{n}$.

Proposition 3.6. Let $P$ be a binomial poset of rank $n$ with factorial function $B(k)=k !$ for $1 \leq k \leq n$. Then the poset $P$ is isomorphic to the boolean lattice $B_{n}$ of rank $n$.

The following is [4, Lemma 2.12].

Lemma 3.7. Let $P^{\prime}$ and $P$ be two Eulerian binomial posets of rank $2 m+2, m \geq 2$, having atom functions $A^{\prime}(n)$ and $A(n)$, respectively, which agree for $n \leq 2 m$. Then the following equality holds:

$$
\frac{1}{A(2 m+1)}\left(1-\frac{1}{A(2 m+2)}\right)=\frac{1}{A^{\prime}(2 m+1)}\left(1-\frac{1}{A^{\prime}(2 m+2)}\right) .
$$


Lemma 3.8. Every Eulerian binomial poset $P$ of rank 4 is isomorphic to either $T_{4}$ or $B_{4}$.

In the following theorem we obtain the structure of Eulerian binomial posets of even rank.

Theorem 3.9. Every Eulerian binomial poset of even rank $n=2 m \geq 4$ is isomorphic to either $T_{n}$ or $B_{n}$ (the butterfly poset of rank $n$ or boolean lattice of rank $n$ ).

Theorem 3.10. Let $P$ be an Eulerian binomial poset of odd rank $n=2 m+1 \geq 5$. Then the poset $P$ satisfies one of the following conditions:

(i) There is a positive integer $k$ such that $P$ is the $k$-summation of the boolean lattice of rank $n$. In other words, $P \cong \boxplus^{k}\left(B_{n}\right)$.

(ii) There is a positive integer $k$ such that $P$ is the $k$-summation of the butterfly poset of rank $n$. In other words, $P \cong \boxplus^{k}\left(T_{n}\right)$.

Proof. We prove the theorem for two different cases $B(3)=4$ and $B(3)=6$. Lemma 3.8 implies that every interval of length 4 is isomorphic either to $B_{4}$ or $T_{4}$. Thus the factorial function $B(3)$ can only take the values 4 or 6 and therefore we are in one of these two cases.

1. $B(3)=6$. In this case we claim that there is a positive integer $k$ such that $P \cong \boxplus^{k}\left(B_{n}\right)$. In order to show that $P \cong \boxplus^{k}\left(B_{n}\right)$, we make the following construction. We remove $\hat{1}$ and $\hat{0}$ from $P$. The remaining poset is a disjoint union of connected components. Consider one of the obtained connected components and add a minimal element $\hat{0}$ and a maximal element $\hat{1}$ to it. Denote the resulting poset by $Q$. We wish to show that $Q \cong B_{n}$. This implies that $P \cong \boxplus^{k}\left(B_{n}\right)$. It is not hard to see that $Q$ is an Eulerian binomial poset. The posets $P$ and $Q$ have the same factorial functions and atom functions up to rank $2 m$. Hence $B_{Q}(k)=B_{P}(k)$ and $A_{Q}(k)=A_{P}(k)$, for $1 \leq k \leq 2 m$. Therefore, Eq. (2) implies that the number of atoms and coatoms are the same in the poset $Q$. Denote this number by $t$. Let $x_{1}, \ldots, x_{t}$ and $a_{1}, \ldots, a_{t}$ be an ordering of the atoms and coatoms of $Q$, respectively. Also, let $c_{1}, \ldots, c_{l}$ be the set of elements of rank $2 m-1$ in $Q$. We show that $t=2 m+1$, and this implies that $Q \cong B_{2 m+1}$. For each element $y$ of rank at least 2 in $Q$, let $S(y)$ be the set of atoms of $Q$ that are below $y$. Set $A_{i}:=S\left(a_{i}\right)$ for each element $a_{i}$ of rank $2 m, 1 \leq i \leq t$, and also set $C_{i}:=S\left(c_{i}\right)$ for each element $c_{i}$ of rank $2 m-1,1 \leq i \leq l$. In order to show that $Q \cong B_{n}$, we prove the following.

(1) We show that $\left|A_{i} \cap A_{j}\right|=2 m-1$ for $i \neq j$.

(2) We use part (1) to show that $t=2 m+1$.

(1) We first show that $\left|A_{i} \cap A_{j}\right|=2 m-1$ for $i \neq j$. By considering the factorial functions, Theorem 3.9 implies that the intervals $\left[\hat{0}, a_{i}\right]$ and $\left[x_{j}, \hat{1}\right]$ have the same factorial functions as $B_{2 m}$ and so they are isomorphic to $B_{2 m}$ for

$1 \leq i \leq t$ and $1 \leq j \leq t$. We conclude that any interval $\left[\hat{0}, c_{k}\right]$ of rank $2 m-1$ is isomorphic to $B_{2 m-1}$. As a consequence, we have $\left|A_{i}\right|=\left|S\left(a_{i}\right)\right|=2 m, 1 \leq i \leq t$ and also $\left|C_{k}\right|=$ $\left|S\left(c_{k}\right)\right|=2 m-1,1 \leq k \leq l$.

If there exist $i$ and $j$ such that $A_{i} \cap A_{j} \neq \emptyset$, where $1 \leq i, j \leq t$, we claim that $2 m-1 \leq$ $\left|A_{i} \cap A_{j}\right| \leq 2 m$. Consider an atom $x_{k} \in A_{i} \cap A_{j}, 1 \leq k \leq t$. Theorem 3.9 implies that 
$\left[x_{k}, \hat{1}\right] \cong B_{2 m}$. Thus, by considering properties of boolean lattices, there is an element $c_{h}$ of rank $2 m-2$ in this interval which is covered by $a_{i}$ and $a_{j}, 1 \leq h \leq l$. Notice that $c_{h}$ is an element of rank $2 m-1$ in $Q$. Therefore, $\left|C_{h}\right|=2 m-1 \leq\left|A_{i} \cap A_{j}\right| \leq\left|A_{i}\right|=\left|S\left(a_{i}\right)\right|=2 m$. We claim that for all distinct pairs $i$ and $j, 1 \leq i, j \leq t$, we have $A_{i} \cap A_{j} \neq \emptyset$. In order to show this claim, associate the graph $G_{Q}$ to the poset $Q$ as follows: $A_{1}, \ldots, A_{t}$ are vertices of this graph, and we connect vertices $A_{i}$ and $A_{j}$ if and only if $A_{i} \cap A_{j} \neq \emptyset$.

We will show that $G_{Q}$ is a complete graph and so $\left|A_{i} \cap A_{j}\right| \neq 0$ for all $i \neq j$. Since $Q-\{\hat{0}, \hat{1}\}$ is connected, $G_{Q}$ is also a connected graph. We show that if $\left\{A_{i}, A_{j}\right\}$ and $\left\{A_{j}, A_{k}\right\}$ are different edges of $G_{Q},\left\{A_{i}, A_{k}\right\}$ is also an edge of $G_{Q}$. Since $\left\{A_{i}, A_{j}\right\}$ and $\left\{A_{j}, A_{k}\right\}$ are edges of $G_{Q}$, we have $\left|A_{i} \cap A_{j}\right| \geq 2 m-1$ as well as $\left|A_{j} \cap A_{k}\right| \geq 2 m-1$. On the other hand, since $\left|A_{i}\right|=\left|A_{j}\right|=\left|A_{k}\right|=2 m$, we conclude that $A_{i} \cap A_{k} \neq \emptyset$. Therefore $\left\{A_{i}, A_{k}\right\}$ is also an edge of $G_{Q}$. As a consequence, the connected graph $G_{Q}$ is a complete graph. Thus $A_{i} \cap A_{j} \neq \emptyset$ and also $2 m-1 \leq\left|A_{i} \cap A_{j}\right| \leq 2 m$ for $1 \leq i, j \leq t$ and $i \neq j$.

Now, we show that $\left|A_{i} \cap A_{j}\right|=2 m-1$ for all $i \neq j$. We proceed by contradiction. Suppose this claim does not hold. Then there are different $i$ and $j$ such that $\left|A_{i} \cap A_{j}\right|=2 m$. We claim that in the case $\left|A_{i} \cap A_{j}\right|=2 m$, there are two elements of rank $2 m-1$ in $Q$ such that they both are covered by coatoms $a_{i}$ and $a_{j}$. To show this claim, consider an atom $x_{f} \in A_{i} \cap A_{j}$, so we have $\left[x_{f}, \hat{1}\right] \cong B_{2 m}$. Hence, there is a unique element $c_{h}$ of rank $2 m-2$ in this interval which is covered by both $a_{i}$ and $a_{j}$. By induction on $m$, Lemma 3.4, and the property that $\left|C_{h}\right| \leq\left|A_{i} \cap A_{j}\right|=2 m$, we conclude that $\left[\hat{0}, c_{h}\right]$ is isomorphic to $B_{2 m-1}$ and so $\left|C_{h}\right|=2 m-1$. Therefore there is an atom $x_{d} \in A_{i} \cap A_{j} \backslash C_{h}$. Since the interval $\left[x_{d}, \hat{1}\right]$ is isomorphic to $B_{2 m}$, there is an element $c_{k} \neq c_{h}$ of rank $2 m-1$ which is covered by coatoms $a_{i}$ and $a_{j}$.

Since $\left|C_{h}\right|=\left|S\left(c_{h}\right)\right|=\left|C_{k}\right|=\left|S\left(c_{k}\right)\right|=2 m-1$ and $C_{k}$ and $C_{h}$ are both subsets of $A_{i} \cap A_{j}$, we conclude that there should be an atom $x_{s} \in C_{k} \cap C_{h}$. Therefore the interval $\left[x_{s}, \hat{1}\right]$ has two elements $c_{k}$ and $c_{h}$ of rank $2 m-2$ such that they both are covered by two elements $a_{i}$ and $a_{j}$ of rank $2 m-1$ in the interval $\left[x_{s}, \hat{1}\right]$. We know $\left[x_{s}, \hat{1}\right] \cong B_{2 m}$ and there are no two elements of rank $2 m-2$ covered by two elements of rank $2 m-1$ in $B_{2 m}$. This contradicts our assumption, and so $\left|A_{i} \cap A_{j}\right|=2 m-1$ for pairs $i$ and $j$ of distinct elements.

In summary, we have:

(a) $\left|A_{i}\right|=2 m$ for $1 \leq i \leq t$,

(b) $\left|A_{i} \cap A_{j}\right|=2 m-1$ for all $1 \leq i<j \leq t$,

(c) $\bigcup_{i=1}^{t} A_{i}=\left\{x_{1}, \ldots, x_{t}\right\}$.

As a consequence, we have $t>2 m$.

(2) Now, we show that $t=2 m+1$. We are going to show that $t=2 m+1$. Without loss of generality, consider the three different sets $A_{1}=S\left(a_{1}\right), A_{2}=S\left(a_{2}\right)$ and $A_{3}=S\left(a_{3}\right)$ associated with the three coatoms $a_{1}, a_{2}$ and $a_{3}$. We know that $\left|A_{1}\right|=\left|A_{2}\right|=\left|A_{3}\right|=2 \mathrm{~m}$ and $\left|A_{1} \cap A_{2}\right|=\left|A_{2} \cap A_{3}\right|=\left|A_{1} \cap A_{3}\right|=2 m-1$. Without loss of generality, let us assume that $A_{1}=\left\{x_{1}, x_{2}, \ldots, x_{2 m-1}, y_{1}\right\}$ and $A_{2}=\left\{x_{1}, x_{2}, \ldots, x_{2 m-1}, y_{2}\right\}$ where $y_{i} \neq$ $x_{1}, \ldots, x_{2 m-1}$ for $i=1,2$. We have the following two different cases:

(a) $A_{3}$ does not contain $y_{1}$ and $y_{2}$. 
(b) $A_{3}$ contains at least one of $y_{1}$ and $y_{2}$.

First we study the case, $A_{3}=\left\{x_{1}, x_{2}, \ldots, x_{2 m-1}, y_{3}\right\}$ where $y_{3} \notin\left\{y_{1}, y_{2}, x_{1}, \ldots, x_{2 m-1}\right\}$. Considering the $t-3$ other coatoms $a_{k}, 4 \leq k \leq t$, there are different atoms $y_{k}, 4 \leq k \leq t$, such that $y_{k} \notin\left\{y_{1}, y_{2}, y_{3}, x_{1}, \ldots, x_{2 m-1}\right\}$ and $A_{k}=S\left(a_{k}\right)=\left\{x_{1}, x_{2}, \ldots, x_{2 m-1}, y_{k}\right\}$. This implies that the number of atoms is $\left|\bigcup_{i=1}^{t} A_{i}\right|=t+2 m-1$, which is a contradiction. So it must be the case that $A_{3}$ contains one of $y_{1}$ or $y_{2}$. In this case $\left|A_{2} \cap A_{3}\right|=\left|A_{1} \cap A_{3}\right|=2 m-1$ implies that $A_{3}=\left\{x_{1}, x_{2}, \ldots, x_{2 m-1}, y_{1}, y_{2}\right\} \backslash\left\{x_{j}\right\} \subset A_{1} \cup A_{2}$ for some $x_{j}$. Since $A_{3}$ was chosen arbitrarily, it follows that for each $A_{k}$ we have $A_{k} \subset A_{1} \cup A_{2}$.

Therefore,

$$
\bigcup_{i=1}^{t} A_{k}=\left\{x_{1}, \ldots, x_{2 m-1}, y_{1}, y_{2}\right\}
$$

Thus the number of coatoms in the poset $Q$ is $t=2 m+1$.

By Theorem 3.9. $B_{Q}(k)=k$ ! for $1 \leq k \leq 2 m$, therefore $B_{Q}(2 m+1)=(2 m+1)$ !. By Proposition 3.6, $Q$ is isomorphic to $B_{2 m+1}$ and so $P$ is a union of copies of $B_{2 m+1}$ with their minimal elements and maximal elements identified. In other words, $P \cong \boxplus^{k}\left(B_{2 m+1}\right)$. It can be seen that $P$ is binomial and Eulerian and the proof follows.

(ii) $B(3)=4$. With the same argument as part $(i)$, we remove $\hat{1}$ and $\hat{0}$ from $P$. The remaining poset is a disjoint union of connected components. We add a minimal element $\hat{0}$ and a maximal element $\hat{1}$ to each of these connected components. We show that the obtained posets are isomorphic to $T_{n}$. This implies that $P \cong \boxplus^{k}\left(T_{n}\right)$.

We construct the binomial poset $Q$ by adding $\hat{1}$ and $\hat{0}$ to one of the connected components of $P-\{\hat{0}, \hat{1}\}$. We claim that $Q$ is isomorphic to $T_{2 m+1}$. Similar to part $(i)$, let $a_{1}, \ldots, a_{t}$ and $x_{1}, \ldots, x_{t}$ denote coatoms and atoms of $Q$. We show that $t=2$ which implies $Q \cong T_{2 m+1}$.

Set $A_{i}=S\left(a_{i}\right)$. By Theorem 3.9. we have $\left|A_{i}\right|=2$. It is easy to see that $\bigcup_{i=1}^{t} A_{i}=\left\{x_{1}, \ldots, x_{t}\right\}$. Define $G_{Q}$ to be the graph with vertices $x_{1}, \ldots, x_{t}$ and edges $A_{1}, \ldots, A_{t}$. Since $Q \backslash\{\hat{0}, \hat{1}\}$ is connected, $G_{Q}$ is also a connected graph. Since $\left[x_{i}, \hat{1}\right] \cong T_{2 m}$, the degree of each vertex of $G_{Q}$ is 2 and $G_{Q}$ is the cycle of length $t$. Therefore if $t>2$, we have $\left|A_{i} \cap A_{j}\right|=1$ or $0,1 \leq i<j \leq t$.

We claim that $t=2$. Suppose this claim does not hold and $t>2$. Consider an element $c$ of rank 3 in $Q$. Lemma 3.4 and Theorem 3.9 imply that both intervals $[\hat{0}, c]$ and $[c, \hat{1}]$ are isomorphic to butterfly posets. Hence there are two coatoms above $c$, say $a_{k}$ and $a_{l}$, and similarly there are two atoms below $c$, say $x_{h}$ and $x_{s}$. Therefore, we have $A_{k}=A_{l}=\left\{x_{h}, x_{s}\right\}$. This is not possible when $t>2$. As a consequence, $t=2$ and all the $A_{i}$ 's have two elements and $\left|\bigcup_{1}^{t} A_{i}\right|=\left|\left\{x_{1}, \ldots, x_{t}\right\}\right|=2=t$. Similar to part $(i), B_{Q}(k)=2^{k-1}$ for $1 \leq k \leq 2 m+1$. By Proposition 3.5 . we conclude that $Q$ is isomorphic to $T_{2 m+1}$. Therefore, there is an integer $k>0$ such that $P \cong \boxplus^{k}\left(T_{n}\right)$.

\section{Finite Eulerian Sheffer Posets}

In this section, we give an almost complete classification of the factorial functions and the structure of Eulerian Sheffer posets. We study Eulerian Sheffer posets of ranks $n=3$ and 4 in Lemmas 3.4 and 4.2 . 
By these two lemmas, we reduce the set of possible values of $B(3)$ to 4 or 6 . In Section 4.1 Lemma 4.3 and Theorems 4.4, 4.9, 4.10 and 4.11 deal with Eulerian Sheffer posets with $B(3)=6$. Finally in Section 4.2. Theorem 4.12 deals with Eulerian Sheffer posets with $B(3)=4$.

It is clear that every binomial poset is also a Sheffer poset. Here is an other example of Sheffer posets, some of which appear in [4] and [9].

Example 4.1. Let $T$ be the poset with the elements $\hat{0}_{1}, \hat{0}_{2}, \hat{1}$ and the cover relations $\hat{0}_{1}<\hat{1}$ and $\hat{0}_{2}<\hat{1}$.

Let $T^{n}$ be the Cartesian product of $n$ copies of the poset $T$. The poset $C_{n}=T^{n} \cup\{\hat{0}\}$ denotes the face lattice of an n-dimensional cube, also known as the cubical lattice. The cubical lattice is a Sheffer poset with $B(k)=k$ ! for $1 \leq k \leq n$ and $D(k)=2^{k-1}(k-1)$ ! for $1 \leq k \leq n+1$.

It is not hard to see that Lemma 3.4 also characterize the structure of Eulerian Sheffer posets of rank 3. Lemma 4.2 deals with Eulerian Sheffer posets of rank 4.

Lemma 4.2. Let poset $P$ be an Eulerian Sheffer poset of rank 4. Then one of the following conditions hold.

1. $B(3)=2 b, D(3)=4, D(4)=4 b$, where $b \geq 2$.

2. $B(3)=8, D(3)=3$ !, $D(4)=2^{3} \cdot 3$ !.

3. $B(3)=10, D(3)=3$ !, $D(4)=5$ !.

4. $B(3)=4, D(3)=3 !, D(4)=2 \cdot 3$ !.

5. $B(3)=3 !, D(3)=3 !, D(4)=4$ !

6. $B(3)=3$ !, $D(3)=4, D(4)=2 \cdot 3$ !.

7. $B(3)=3$ !, $D(3)=10, D(4)=5$ !.

8. $B(3)=3$ !, $D(3)=8, D(4)=2^{3} \cdot 3$ !.

9. $B(3)=4, D(3)=2 b, D(4)=4 b$ where $b \geq 2$.

\subsection{Characterization of the factorial functions and structure of Eulerian Sheffer posets of rank $n \geq 5$ for which $B(3)=3$ !.}

In this subsection. we mainly consider Eulerian Sheffer posets with $B(3)=3$ !. As a consequence of Lemma 4.2. we know that Eulerian Sheffer posets of rank $n \geq 4$ with $B(3)=3$ ! have the Sheffer factorial functions $D(3)=4,6,8$ and 10. Lemma 4.3 shows that for any such poset of rank $n \geq 6$, the Sheffer factorial function $D(3)$ can only take the values 4,6 or 8 .

In Subsections 4.1.1 4.1.2 and 4.1.3, we consider posets with $B(3)=6$ and different cases $D(3)=4,6$ and 8 , respectively. The question of studying the finite Eulerian Sheffer posets of rank 5 with $B(3)=6$ and $D(3)=10$ remains open. There is such a poset, namely the face lattice of the 120-cell with Schläfli symbol $\{5,3,3\}$.

Lemma 4.3. Let $P$ be an Eulerian Sheffer poset of rank $n \geq 6$ with $B(3)=3$ !. Then $D(3)$ can take only the values $4,6,8$. 


\subsubsection{Characterization of the factorial functions of Eulerian Sheffer posets of rank $n \geq 5$ for which $B(3)=3$ ! and $D(3)=8$.}

In this subsection, we study the factorial functions of Eulerian Sheffer posets of rank $n \geq 5$ for which $B(3)=3$ ! and $D(3)=8$. Theorem 4.4 characterizes the factorial functions of such posets of even rank. However, the question of characterizing the factorial functions of Eulerian Sheffer posets of odd rank $n=2 m+1 \geq 5$ with $B(3)=3$ ! and $D(3)=8$ remains open.

Theorem 4.4. Let $P$ be an Eulerian Sheffer poset of even rank $n=2 m+2 \geq 4$ with $B(3)=3$ ! and $D(3)=8$. Then $P$ has the same factorial functions as $C_{n}$, the cubical lattice of rank $n$, that is, $D(k)=2^{k-1}(k-1) !, 1 \leq k \leq n$ and $B(k)=k !, 1 \leq k \leq n-1$.

In order to prove Theorem 4.4, we establish the following two lemmas.

Lemma 4.5. Let $Q$ be an Eulerian Sheffer poset of odd rank $2 m+1, m \geq 2$, with $B(3)=3$ !. Then the coatom function of $Q$ must satisfy at least one of the following inequalities: $C(n) \neq 2(n-1)$ for $2 \leq n \leq 2 m$ and $C(2 m+1) \neq 4 m+1$.

Lemma 4.5 implies the following.

Corollary 4.6. Let $P$ be an Eulerian Sheffer poset of rank $2 m+2, m \geq 2$, with $B(k)=k$ !, for $1 \leq k \leq 2 m$. Then the coatom function of $P$ must satisfy at least one of the following inequalities: $C(n) \neq 2(n-1), 2 \leq n \leq 2 m, C(2 m+1) \neq 4 m+1$ and $C(2 m+2) \neq 4(2 m+1)$.

Lemma 4.7. Let $Q$ be an Eulerian Sheffer poset of rank $2 m+2, m \geq 2$, with $B(k)=k !$ for $1 \leq k \leq 2 m$. Then the coatom function of $Q$ must satisfy at least one of the following inequalities: $C(n) \neq 2(n-1)$, $2 \leq n \leq 2 m, C(2 m+1) \neq 4 m-1$ and $C(2 m+2) \neq \frac{4}{3}(2 m+1)$.

The following lemma can be obtained by applying the proof of Lemma 4.8 in [4].

Lemma 4.8. Let $P$ and $P^{\prime}$ be two Eulerian Sheffer posets of rank $2 m+2, m \geq 2$, such that their binomial factorial functions and coatom functions agree up to rank $n \leq 2 m$. That is, $B(n)=B^{\prime}(n)$ and $C(n)=C^{\prime}(n)$, where $m \geq 2$. Then the following equation holds:

$$
\frac{1}{C(2 m+1)}\left(1-\frac{1}{C(2 m+2)}\right)=\frac{1}{C^{\prime}(2 m+1)}\left(1-\frac{1}{C^{\prime}(2 m+2)}\right) .
$$

of Theorem 4.4 In order to prove the theorem, we inductively show that the Eulerian Sheffer poset $P$ and $C_{2 m+2}$, the cubical latice of rank $2 m+2$, have the same coatom functions.

Let $C(k)$ and $C^{\prime}(k)=2(k-1)$ respectively be the coatom functions of the Eulerian Sheffer poset $P$ and $C_{2 m+2}$ for $2 \leq k \leq 2 m+2$. We only need to show that $C(k)=C^{\prime}(k)=2(k-1)$ for $2 \leq k \leq 2 m+2$. We prove this claim by induction on $m$. By Lemma 4.2, an Eulerian Sheffer poset of even rank 4 with $B(3)=3$ ! and $D(3)=8$ has the same factorial function as $C_{4}$. Therefore, $C(4)=C^{\prime}(4)=6$ and the claim holds for $m=1$. Suppose $m \geq 2$. By the induction hypothesis $C(k)=C^{\prime}(k)=2(k-1)$ for $2 \leq k \leq 2 m$. Set $F=C(2 m+1)$ and $E=C(2 m+2)$. Theorem 3.10 implies that $B(k)=k$ ! for $1 \leq k \leq 2 m$ and there is a positive integer $\alpha$ such that $B(2 m+1)=\alpha(2 m+1)$ !. We know that $D(k)=2^{k-1}(k-1)$ ! for $1 \leq k \leq 2 m$, so $D(2 m+1)=F 2^{2 m-1}(2 m-1)$ ! and $D(2 m+2)=$ $E F 2^{2 m-1}(2 m-1)$ !. Since $P$ is an Eulerian Sheffer poset, the Euler-Poincaré relation implies that

$$
1+\sum_{k=1}^{2 m+2} \frac{(-1)^{k} D(2 m+2)}{D(k) B(2 m+2-k)}=0
$$


By substituting the values of the factorial functions, we have

$$
2-E+\frac{E F}{2}\left[\frac{1}{2 m}-\frac{1}{2 m(2 m+1)}+\frac{2^{2 m}}{2 m(2 m+1)}-\frac{2^{2 m}}{2 \alpha m(2 m+1)}\right]=0
$$

Thus,

$$
E\left(1-F\left(\frac{2 \alpha m+(\alpha-1) 2^{2 m}}{4 \alpha m(2 m+1)}\right)\right)=2 .
$$

In case $\alpha \geq 2$, it is easy to verify that

$$
\left(\frac{2 \alpha m+(\alpha-1) 2^{2 m}}{4 \alpha m(2 m+1)}\right)>\frac{1}{2 m}
$$

Since $F \geq A(2 m) \geq 2 m$, the left-hand side of Eq. 9 becomes negative in this case. Therefore, $\alpha=1$ and the posets $P$ and $C_{2 m+2}$ have the same binomial factorial functions. Since $2 m+1=A(2 m+1) \leq$ $C(2 m+2)<\infty$, Lemma 4.8 implies that $4 m-1 \leq C(2 m+1)=F \leq 4 m+1$. Since $\alpha=1$, Eq. (9) implies that $2-E+\frac{E F}{4 m+2}=0$. Thus $E$ and $F$ must satisfy one of the following cases:

(1) $F=4 m-1$ and $E=\frac{4}{3}(2 m+1)$.

(2) $F=4 m$ and $E=4 m+2$.

(3) $F=4 m+1$ and $E=4(2 m+1)$.

As we have discussed in Corollary 4.6 and Lemma 4.7, the cases (1) and (3) are not possible. Case (2) occurs in the cubical lattice of rank $2 m+2, C_{2 m+2}$. Thus, the poset $P$ has the same factorial functions as $C_{2 m+2}$, as desired.

Classification of the factorial functions of Eulerian Sheffer posets of odd rank $n=2 m+1 \geq 5$ with $B(3)=6$ and $D(3)=8$ remains open. Let $\alpha$ be a positive integer and set $Q_{\alpha}=\boxplus^{\alpha}\left(C_{2 m+1}\right)$. It can be seen that $Q_{\alpha}$ is an Eulerian Sheffer poset and it has the following factorial functions: $D(k)=2^{k-1}(k-1)$ ! for $1 \leq k \leq n-1, D(n)=\alpha \cdot 2^{n-1}(n-1)$ ! and $B(k)=k$ ! for $1 \leq k \leq n-1$. We ask the following question:

Question: Let $P$ be an Eulerian Sheffer poset of odd rank $n=2 m+1 \geq 5$ with $B(3)=6, D(3)=$ 8. Is there a positive integer $\alpha$ such that the poset $P$ has the same factorial functions as poset $Q_{\alpha}=$ $\boxplus^{\alpha}\left(C_{2 m+1}\right)$, where $C_{2 m+1}$ is a cubical lattice of rank $2 m+1$ ?

\subsubsection{Characterization of the structure of Eulerian Sheffer posets of rank $n \geq 5$ for which $B(3)=3$ !, and $D(3)=3$ ! $=6$.}

Theorem 4.9. Let $P$ be an Eulerian Sheffer poset of rank $n \geq 3$ with $B(3)=D(3)=3$ ! $=6$ for 3-intervals. $P$ satisfies one of the following cases:

(i) There is an integer $k \geq 1$ such that $P \cong \boxplus^{k}\left(B_{n}\right)$, where $n$ is odd.

(ii) $P \cong B_{n}$, where $n$ is even. 


\subsubsection{Characterization of the structure of Eulerian Sheffer posets of rank $n \geq 5$ for which $B(3)=3$ ! and $D(3)=4$.}

Let $P$ be an Eulerian Sheffer poset of rank $n \geq 5$, with $B(3)=3$ ! and $D(3)=4$. In this section we show that in the case $n=2 m+2$ the poset $P$ satisfies $P \cong \Sigma^{*}\left(\boxplus^{\alpha}\left(B_{2 m+1}\right)\right)$ for some integer $\alpha \geq 1$ and in the case $n=2 m+1, P \cong \boxplus^{\alpha}\left(\Sigma^{*}\left(B_{2 m}\right)\right)$, for some integer $\alpha \geq 1$.

Theorem 4.10. Let $P$ be an Eulerian Sheffer poset of even rank $n=2 m+2 \geq 4$ with $B(3)=3$ ! and $D(3)=4$. Then $P \cong \Sigma^{*}\left(\boxplus^{\alpha}\left(B_{2 m+1}\right)\right)$, where $\alpha=\frac{B(2 m+1)}{(2 m+1) !}$ is a positive integer for $n \geq 6$ and $\alpha=1$ for $n=4$. Consequently the poset $P$ has the following binomial and Sheffer factorial functions.

(i) $B(k)=k$ ! for $1 \leq k \leq 2 m$, and $B(2 m+1)=\alpha(2 m+1)$ !,

(ii) $D(1)=1, D(k)=2(k-1)$ ! for $2 \leq k \leq 2 m+1$, and $D(2 m+2)=2 \alpha(2 m+1)$ !.

Theorem 4.11. Let $P$ be an Eulerian Sheffer poset of odd rank $n=2 m+1 \geq 5$ with $B(3)=6$ and $D(3)=4$. Then $P \cong \boxplus^{\alpha}\left(\Sigma^{*}\left(B_{2 m}\right)\right)$ for some positive integer $\alpha$.

\subsection{Characterization of the structure and factorial functions of Eulerian Sheffer posets of rank $n \geq 5$ with $B(3)=4$.}

In this section, we characterize Eulerian Sheffer posets of rank $n \geq 5$ with $B(3)=4$. Let $P$ be an Eulerian Sheffer poset of rank $n \geq 5$ with $B(3)=4$. It can be seen that the poset $P$ satisfies one of the cases:

1. $P$ has the following binomial factorial function $B(k)=2^{k-1}$, where $1 \leq k \leq n-1$;

2. $n$ is even and there is a positive integer $\alpha>1$ such that poset $P$ has the binomial factorial function $B(k)=2^{k-1}$ for $1 \leq k \leq n-2$ and $B(n-1)=\alpha \cdot 2^{n-2}$ for some positive integer $\alpha$.

As a consequence of Theorems 3.11 and 3.12 in [4], we can characterize posets in the case $(i)$. Theorem 4.12 deals with the case $(i i)$.

Theorem 4.12. Let $P$ be an Eulerian Sheffer poset of even rank $n=2 m+2>4$ with the binomial factorial function $B(k)=2^{k-1}$ for $1 \leq k \leq 2 m$, and $B(2 m+1)=\alpha \cdot 2^{2 m}$, where $\alpha>1$ is a positive integer. Then $P \cong \Sigma^{*}\left(\boxplus^{\alpha}\left(T_{2 m+1}\right)\right)$.

\section{References}

[1] J. Backelin, Binomial posets with non-isomorphic intervals, math.CO/0508397, 22 August, 2005.

[2] A. Björner, V. Welker, Segre and Rees products of posets, with ring-theoretic applications, J. Pure Appl. Algebra 198 (2005) 43-55.

[3] P. Doubilet, G.-C.Rota, R. Stanley, On the foundations of combinatorial theory (VI). The idea of generating functions, in Sixth Berkeley Symp. on Math. Stat. and Prob., vol. 2: Probability Theory, Univ. of California, Berkeley, 1972, pp. 267-318.

[4] R. Ehrenborg and M. Readdy, Classification of the factorial functions of Eulerian binomial and Sheffer posets, J. Combin. Theory Ser. A 114 (2007) 339-359. 
[5] R. Ehrenborg and M. Readdy, Sheffer posets and r-signed permutations, Ann. Sci. Math. Québec 19 (1995) 173-196.

[6] J. Farley and S. Schmidt, Posets that locally resemble distributive lattices, J. Combin. Theory Ser. A 92 (2000) 119-137.

[7] D.J. Grabiner, Posets in which every interval is a product of chains, and natural local actions of the symmetric group, Discrete Math. 199 (1999) 77-84.

[8] G. Hetyei, Matrices of formal power series associated to binomial posets. J. Algebraic Combin. 22 (2005) 65-104.

[9] V. Reiner, Upper binomial posets and signed permutation statistics, European J. Combin. 14 (1993) 581-588.

[10] R. Simion, R. Stanley, Flag-symmetry of the poset of shuffles and a local action of the symmetric group, Discrete Math. 204 (1999) 369-396.

[11] R. Stanley, Binomial posets, Möbius inversion, and permutation enumeration, J. Combin. Theory Ser. A 20 (1976) 336-356.

[12] R. Stanley, Enumerative Combinatorics, vol. I, Wadsworth and Brooks/Cole, Pacific Grove, 1986.

[13] R. Stanley, Flag-symmetric and locally rank-symmetric partially ordered sets, Electron. J. Combin. 3 (R6) (1996) 22. 